ARTICLE

\title{
Monolayer-to-bilayer transformation of silicenes and their structural analysis
}

\author{
Ritsuko Yaokawa1, Tetsu Ohsuna1, Tetsuya Morishita², Yuichiro Hayasaka ${ }^{3}$, Michelle J.S. Spencer ${ }^{4}$ \\ \& Hideyuki Nakano ${ }^{1,5}$
}

Silicene, a two-dimensional honeycomb network of silicon atoms like graphene, holds great potential as a key material in the next generation of electronics; however, its use in more demanding applications is prevented because of its instability under ambient conditions. Here we report three types of bilayer silicenes that form after treating calcium-intercalated monolayer silicene $\left(\mathrm{CaSi}_{2}\right)$ with a $\mathrm{BF}_{4}^{-}$-based ionic liquid. The bilayer silicenes that are obtained are sandwiched between planar crystals of $\mathrm{CaF}_{2}$ and/or $\mathrm{CaSi}_{2}$, with one of the bilayer silicenes being a new allotrope of silicon, containing four-, five- and six-membered $s p^{3}$ silicon rings. The number of unsaturated silicon bonds in the structure is reduced compared with monolayer silicene. Additionally, the bandgap opens to $1.08 \mathrm{eV}$ and is indirect; this is in contrast to monolayer silicene which is a zero-gap semiconductor.

\footnotetext{
${ }^{1}$ TOYOTA Central R\&D Labs, Inc., 41-1, Yokomichi, Nagakute, Aichi 480-1192, Japan. ${ }^{2}$ CD-FMat, National Institute of Advanced Industrial Science and Technology (AIST), Central 2, 1-1-1 Umezono, Tsukuba, Ibaraki 305-8568, Japan. ${ }^{3}$ The Electron Microscopy Center, Tohoku University, Katahira 2-1-1, Aobaku, Sendai 980-8577, Japan. ${ }^{4}$ School of Science, RMIT University, GPO Box 2476, Melbourne, Victoria 3001, Australia. ${ }^{5}$ JST Presto, Kawaguchi 332-0012, Japan. Correspondence and requests for materials should be addressed to R.Y. (email: e4777@mosk.tytlabs.co.jp) or to H.N. (email: hnakano@mosk.tytlabs.co.jp).
} 
A frenzy of interest in graphene has spawned many theoretical and experimental studies ${ }^{1-4}$. After calculating the structures of two-dimensional (2D) crystals of silicon (silicene $)^{5-7}$, researchers have speculated that silicon atoms might form graphene-like sheets and have attempted to produce such silicene structures ${ }^{8-12}$. Very recently, Tao et al. ${ }^{13}$ succeeded in fabricating the first silicene transistor, although the device's performance was modest. Nonetheless, the development of much more facile and practical processing methods has remained a challenging issue. The most difficult problem is that silicene grows on specific substrates and is stable only under vacuum conditions $8,9,14,15$. Another issue is that the influence of the substrate cannot be removed; the strong hybridization between $\mathrm{Si}$ and the substrate may stabilize silicene grown on specific substrates $8,14-16$.

In a previous report on calcium-intercalated silicene $\left(\mathrm{CaSi}_{2}\right)$, we observed a massless Dirac-cone band dispersion at the k-point in the Brillouin zone, which was located far from the Fermi level because of the substantial charge transfer from the $\mathrm{Ca}$ atoms to the silicene layers ${ }^{17}$. This result is similar to the previously reported band structures of silicenes deposited on specific substrates ${ }^{9}$ because $\mathrm{CaSi}_{2}$ is a type of Zintl silicide, in which the formal charge is rewritten as $\mathrm{Ca}^{2}+$ and $\mathrm{Si}^{-}$(ref. 18). Therefore, the intrinsic electronic structure of silicene has never been observed. In the calculated results, a van der Waals bonded silicene layer has been deposited on an intact multi-CaF layer $^{19}$. If the $\mathrm{Ca}$ layer of $\mathrm{CaSi}_{2}$ had been exchanged with a $\mathrm{CaF}_{2}$ layer, the influence of the substrate would have been almost completely suppressed. To reduce the influence of external factors on the electronic structure of silicene (for example, from substrates or counter ions) and to increase the stability under ambient condition, we replaced monolayer silicene with bilayer silicene.

The existence of a bilayer silicene structure, whose density of unsaturated silicon bonds is reduced in comparison with monolayer silicene, has been predicted by molecular dynamics (MD) calculations ${ }^{20-27}$. If we could experimentally prepare a similar bilayer silicene, we could then investigate its intrinsic electronic structure. Because of the electron transfer from the calcium cation, the monolayer silicene in $\mathrm{CaSi}_{2}$ is a formally anionic layer ${ }^{17}$ : when the calcium cation becomes electrically neutral, the silicene will not retain its honeycomb structure and will reconstruct to form a more stable structure. Under this supposition, we attempted to segregate the $\mathrm{Ca}$ and $\mathrm{Si}$ phases while maintaining the layer structures by diffusing fluoride $(\mathrm{F})$ atoms, which are more electronegative than $\mathrm{Si}$, into $\mathrm{CaSi}_{2}$; the goal was to form an ionic bond (or interaction) between $\mathrm{Ca}$ and $\mathrm{F}$. In this study, $\mathrm{BF}_{4}$ anion based ionic liquid was used for the origin of fluoride anion.

\section{Results}

Fluoride diffusion into $\mathbf{C a S i}_{2}$. When the $\mathrm{CaSi}_{2}$ crystal (Supplementary Fig. 1) was annealed in $[\mathrm{BMIM}]\left[\mathrm{BF}_{4}\right]$ ionic liquid at $250-300^{\circ} \mathrm{C}$, it was changed to a $\mathrm{CaSi}_{2} \mathrm{~F}_{\mathrm{X}} \quad(0 \leq \mathrm{X} \leq 2.3)$ compound through diffusion of $\mathrm{F}^{-}$, in which the local $\mathrm{F}^{-}$ concentration gradually decreased from the crystal edge to the interior (Fig. 1a,b and Supplementary Fig. 2). As a result, three types of bilayer $\mathrm{Si}$ in a $\mathrm{CaSi}_{2}$ single crystal were obtained by diffusion of $\mathrm{F}^{-}$. Figure $1 \mathrm{c}$, which displays a high-angle annular dark field scanning transmission electron microscopy (HAADF-STEM) image taken of the $\mathrm{CaSi}_{2} \mathrm{~F}_{1.8}$ compound, shows the alternate stacking of planar crystal domains with layer thicknesses of 1-2 nm. The HAADF-STEM imaging provided an atomic-scale $Z$-contrast image ( $Z$ : atomic number) to distinguish the heavier constituent elements ${ }^{28-30}$. STEM-energy-dispersive $\mathrm{X}$-ray spectroscopy (STEM-EDX) elemental mapping identified the bright-contrast crystal domains, which were identified as the $\mathrm{CaF}_{2}$ phase and the dark domains, which were identified as $\mathrm{Si}$ phases (Fig. 1f-j). We determined the crystal structures of the entire planar region in the images of the $\mathrm{CaSi}_{2} \mathrm{~F}_{1.8}$ and $\mathrm{CaSi}_{2} \mathrm{~F}_{2.0}$ compounds shown in Fig. 1c,d, respectively. These planar domains were identified as trilayer $\mathrm{CaF}_{2}$, trilayer $\mathrm{Si}$, bilayer $\mathrm{CaF}_{2}$ and a novel bilayer silicene (denoted as w-BLSi in Fig. 1c,d) that has not been previously predicted by MD calculations ${ }^{20-27}$. Furthermore, two types of bilayer silicenes, one with inversion symmetry (i-BLSi) and one with mirror symmetry (m-BLSi), were recognized in the $\mathrm{CaSi}_{2} \mathrm{~F}_{0.6-1.0}$ composition area (Fig. 1e and Supplementary Fig. 3). The formation of $\mathrm{m}$-BLSi is in accordance with predictions from a previous MD study ${ }^{22}$. The $\mathrm{i}$ - and $\mathrm{m}-\mathrm{BLSi}$ must be adjacent to a pair of $\mathrm{CaF}_{2}$ and $\mathrm{CaSi}_{2}$ crystal layers. The abundance ratio of $\mathrm{i}-\mathrm{BLSi}$ to $\mathrm{m}$-BLSi was $124: 3$ in the observed HAADF-STEM images. Because the calculated energy of i-BLSi was $0.03 \mathrm{eV}$ per atom lower than that of $\mathrm{m}$-BLSi under vacuum, the abundance ratio is qualitatively reasonable. The average size of $\mathrm{w}$-BLSi is $\sim 30 \mathrm{~nm}$, and that of $\mathrm{m}-\mathrm{BLSi}$ is $\sim 10 \mathrm{~nm}$. The size of i-BLSi is greater than $51 \mathrm{~nm}$, which is the maximum size that can be observed by STEM imaging.

Structural determination of w-BLSi. The atomic structure of the bilayer silicene was determined from HAADF-STEM images that were taken with different incident electron beam directions (Fig. 2a-c, Supplementary Fig. 4 and Supplementary Note 1). As shown in Fig. 2d, the bilayer silicene structure had a $2 \mathrm{D}$ translation symmetry and a wavy morphology (hereafter, we refer to the structure as $\mathrm{w}$-BLSi). The w-BLSi structure consists of two silicenes, with alternating chair and boat conformations, that are vertically connected via four-, five- and six-membered rings. Because w-BLSi consists of only $\mathrm{Si}$ atoms exhibiting tetrahedral coordination, the top atom of the five-membered silicon ring possesses unsaturated silicon bonds (dangling bonds). Therefore, compared with those in monolayer silicene and i- (or m-) BLSi, the density of unsaturated silicon bonds in w-BLSi decreased to 25 and 50\%, respectively (Supplementary Fig. 5).

We determined the atomic positions of $\mathrm{w}$-BLSi from highresolution transmission electron microscopy and HAADF-STEM images as accurately as possible (Supplementary Figs 6 and 7, Supplementary Table 1 and Supplementary Note 2). The 2D translation periods of $\mathrm{w}$-BLSi were $a=0.661(2) \mathrm{nm}$ and $b=0.382(3) \mathrm{nm}$, and the two translation axes were normal to each other (Supplementary Fig. 8). The $a$ period of $\mathrm{w}$-BLSi is similar to the triple lattice spacing of $\mathrm{d}_{11-2}$ in $\mathrm{CaF}_{2}(0.223 \mathrm{~nm})$, and the $b$ period is similar to $\mathrm{d}_{-110}$ in $\mathrm{CaF}_{2}(0.386 \mathrm{~nm})$; that is, the difference between $\mathrm{w}$-BLSi and $\mathrm{CaF}_{2}(111)$ is less than the observation error (Supplementary Fig. 9). Because the atomic arrangement of the (111) plane of the $\mathrm{CaF}_{2}$ crystal exhibited threefold symmetry, three equivalent relative rotation angles were observed between w-BLSi and the $\mathrm{CaF}_{2}$ (111) plane (Supplementary Figs 10 and 11). In addition, the angle between the $[01]_{\mathrm{w}-\mathrm{BLSi}}$ and $[11]_{\mathrm{w}-\mathrm{BLSi}}$ directions was almost $60^{\circ}$ (Supplementary Fig. 10, w-BLSi is described in 2D notation, because $2 \mathrm{D}$ can be expressed more simply than three dimensions). Therefore, Figs $1 \mathrm{c}$ and $2 \mathrm{a}$ show the contrast of two different arrangements of bright dots-specifically, the [01] and [11] direction images (Fig. 2e,g) in the w-BLSi regions. In almost all of the observed HAADF-STEM images, w-BLSi always faced the (111) plane of $\mathrm{CaF}_{2}$, and the $\mathrm{F}$ vacancies (red arrows in Fig. 1d) on the $\mathrm{CaF}_{2}$ (111) surface were recognized at special positions associated with the wavy structure of $\mathrm{w}$-BLSi. A w-BLSi was observed to be sandwiched between two $\mathrm{CaF}_{2}$ layers with an F-site surface vacancy of $\sim 0.5$ at the interface (Fig. 1d, Supplementary Figs 7,12-15 and Supplementary Note 3). 

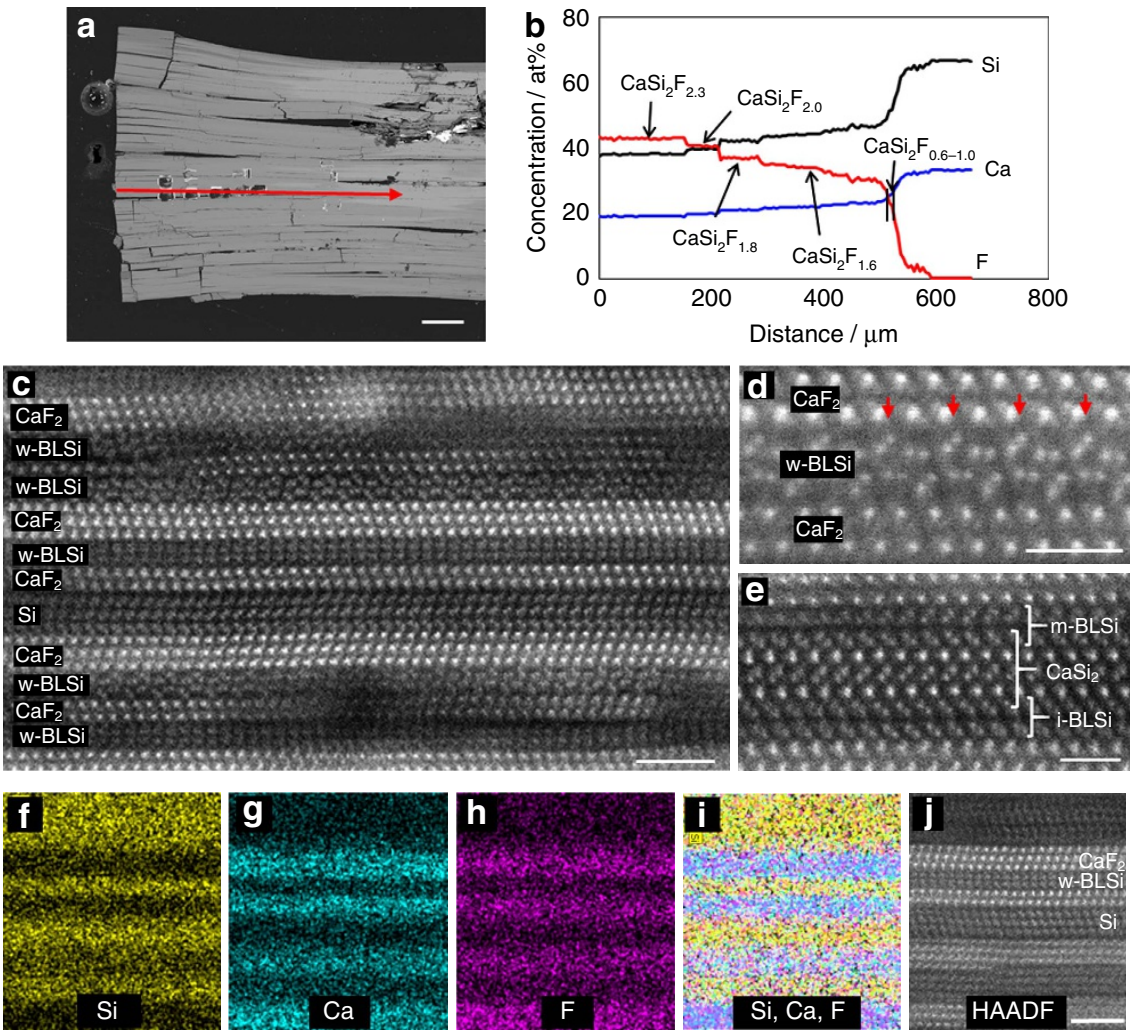

Figure 1 | Visualization of fluoride diffusion. (a) Cross-sectional BSE image of the crystal grain including $\mathrm{CaSi}_{2} \mathrm{~F}_{\mathrm{X}}$ compound. (b) EPMA quantitative line analysis result along the red arrow in a. (c) HAADF-STEM image taken from a region with $\mathrm{CaSi}_{2} \mathrm{~F}_{1.8}$ in $\mathbf{b}$; the strip contrast corresponds to $\mathrm{Si}$ (dark domain) and $\mathrm{CaF}_{2}$ (bright domain) planar crystals. (d) An enlarged HAADF-STEM image taken from a region with $\mathrm{CaSi}_{2} \mathrm{~F}_{2}$ in $\mathbf{b}$; red arrows indicate an F-vacancy site. (e) HAADF-STEM image taken from a region with $\mathrm{CaSi}_{2} \mathrm{~F}_{0.6-1.0}$ in $\mathbf{b}$; bright dots, corresponding to the projected atomic positions of $\mathrm{m}$-and $\mathrm{i}-\mathrm{BLSi}$, can be observed in the image. (f-i) STEM-EDX elemental mapping results of the $\mathrm{CaSi}_{2} \mathrm{~F}_{2}$ composition region. One-element mapping (f: Si; $\mathbf{g}$ : Ca; and $\mathbf{h}$ : F). (i) Overlapped-mapping of Si, Ca and F. (j) HAADF-STEM image of the STEM-EDX elemental mapping area. The scale bars in $\mathbf{a} ; \mathbf{c}$ and $\mathbf{j}$; and $\mathbf{d}$ and $\mathbf{e} ; 100 \mu \mathrm{m}, 2 \mathrm{~nm}$ and $1 \mathrm{~nm}$.

DFT and $a b$ initio MD calculations and optical properties. The w-BLSi structure appears to resemble re-BLSi ${ }^{20}$ in appearance; however, its atomic arrangement is clearly different (Supplementary Fig. 16). An $a b$ initio MD calculation was performed for BLSi under the conditions corresponding to the experimentally observed structure, that is, BLSi was sandwiched between two $\mathrm{CaF}_{2}$ layers with an F-site surface vacancy of 0.5 at the interface. The MD calculation was started with the i-BLSi structure, but it was immediately transformed to another BLSi structure. The system was then equilibrated, and the resultant BLSi structure was found to perfectly agree with the experimentally observed $\mathrm{w}$-BLSi structure in Fig. 3a (Supplementary Tables 2-5, Supplementary Fig. 17 and Supplementary Note 4). The electronic density of states (DOS)

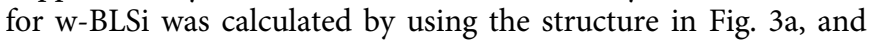
the decomposed DOSs for Si, Ca, and F are shown in Fig. 3b. The $\mathrm{Ca}$ and $\mathrm{F}$ bands are located far below the Fermi level, and the valence bands consist of only Si bands. An ionic rather than a covalent interaction is thus expected between $\mathrm{Si}$ and $\mathrm{Ca}$ or $\mathrm{F}$. We also observe that the bandgap opens to $\sim 0.65 \mathrm{eV}$, in contrast to monolayer silicene, which is a zero-gap semiconductor ${ }^{31}$. Interestingly, however, the gap closes when $\mathrm{w}$-BLSi is isolated without geometry optimization under vacuum (Supplementary Fig. 18b). This result indicates that, in the $\mathrm{CaSi}_{2} \mathrm{~F}_{\mathrm{X}}$ compound, charge transfer from $\mathrm{Ca}$ to $\mathrm{Si}$ occurs, filling the energy levels that are unoccupied under vacuum (Supplementary Discussion). Thus, the electronic properties of $\mathrm{w}$-BLSi appear to be sensitive to its environmental conditions.
The presence of the $\mathrm{F}$ vacancies allows the electrons on $\mathrm{Ca}$ to transfer to $\mathrm{Si}$, which enhances the stability of the w-BLSi structure (Fig. 3a) by saturating the dangling bonds. The $\mathrm{CaF}_{2-\mathrm{x}}$ domains (specifically, ionic crystalline domains) surrounding the Si layers are key to the formation of the w-BLSi structure.

The optical bandgap can be calculated from the absorption spectrum. The diffuse reflectance spectrum of the powder sample with $\mathrm{CaSi}_{2} \mathrm{~F}_{1.8-2.3}$ composition was measured, and the obtained reflectance spectrum data (Supplementary Fig. 19) were converted to a Kubelka-Munk function (K/S), which is proportional to the absorption coefficient $(\alpha)$. The sample was a mixture of $\mathrm{w}$-BLSi, two types of trilayer silicene (with dangling bonds and terminated with F atoms, as shown in Supplementary Fig. 20) and a $\mathrm{CaF}_{2}$ layer (Supplementary Note 5). The relationship between the absorption coefficient $(\alpha)$ and the bandgap energy (Eg) can be described by two types of equations: $\alpha \mathrm{h} v=$ const (direct gap) and $\alpha h v=\mathrm{A}(\mathrm{h} v-\mathrm{Eg})$ (indirect gap), where the DOS for 2D crystals is constant as a function of energy ${ }^{32-35}$ (Supplementary Note 5). Here, h, $v$ and A are Planck's constant, light frequency and proportional constant, respectively. From two linear fittings of the spectrum, the latter equation was found to be suitable for the sample. The absorption edges of the $\mathrm{CaSi}_{2} \mathrm{~F}_{1.8-2.3}$ compound were observed at 1.08 and $1.78 \mathrm{eV}$ (Fig. 3c), assuming indirect transitions.

Freestanding trilayer silicene is semi-metallic, as shown by density functional theory (DFT) calculations ${ }^{36}$. It has been suggested that the bandgap of trilayer silicene with dangling bonds in $\mathrm{CaSi}_{2} \mathrm{~F}_{1.8-2.3}$ is nearly zero if charge transfer between the 


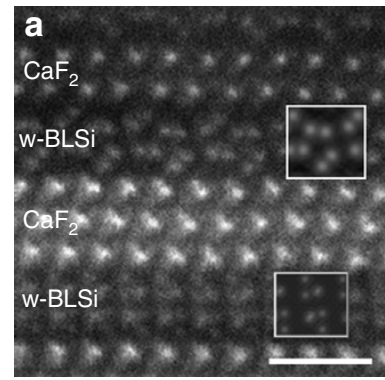

$[01]_{\mathrm{w}-\mathrm{BLSi}}$ and $[11]_{\mathrm{w}-\mathrm{BLSi}}$

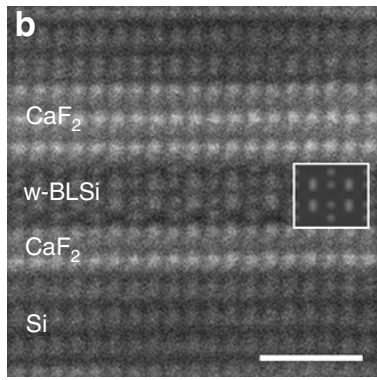

$[10]_{\mathrm{w}-\mathrm{BLSi}}$

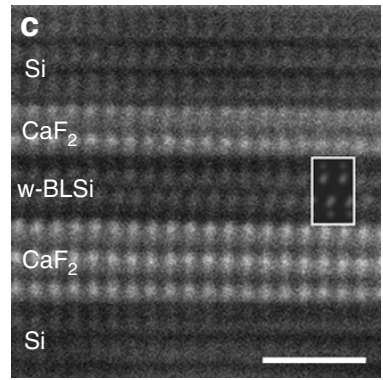

$[13]_{\mathrm{W}-\mathrm{BLSi}}$

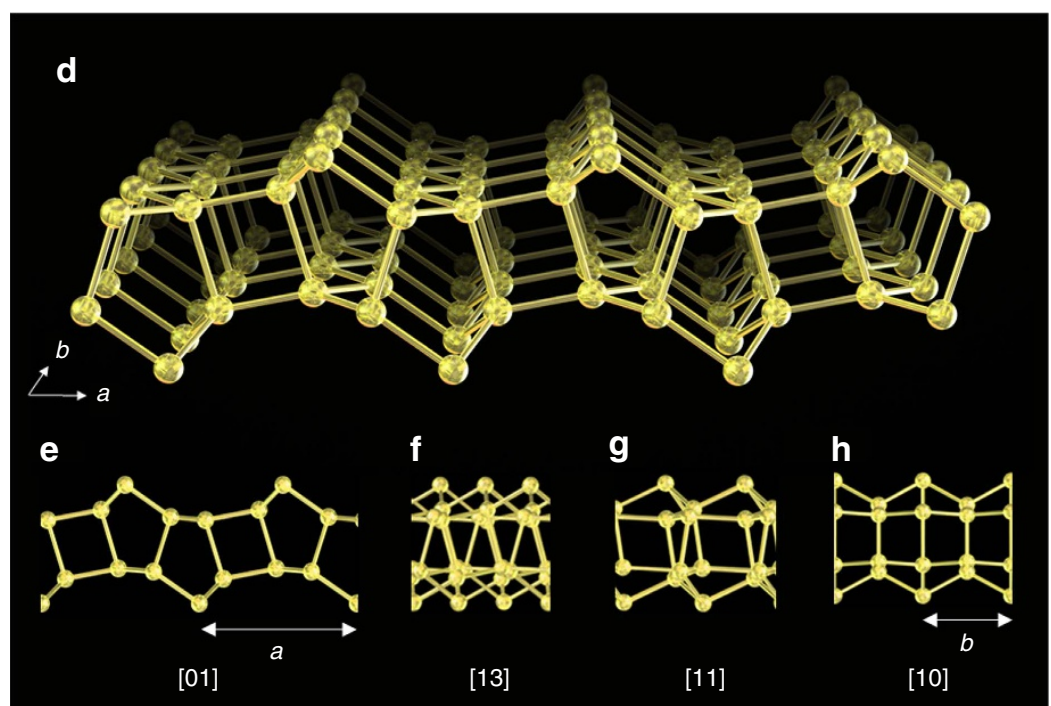

Figure 2 | Structural determination. (a-c) HAADF-STEM and simulation (insets) images of $w-B L S i$. (a) the [01] $]_{W-B L S i}$ and [11] $]_{W-B L S i}$ incident directions $\left([1-10]_{\mathrm{CaF} 2}\right),(\mathbf{b})$ the $[10]_{\mathrm{W}-\mathrm{BLSi}}$ and $[11-2]_{\mathrm{Si}}$ and CaF2 directions and (c) the [13] $]_{\mathrm{W}-\mathrm{BLSi}}$ and $[11-2]_{\mathrm{Si}}$ and CaF2 directions. (d) Schematic illustration of the w-BLSi atomic structure. (e-h) Schematic structures projected in each direction in e [01], f [13], $\mathbf{g}$ [11] and $\mathbf{h}$ [10] directions. All scale bars in (a-c), $1 \mathrm{~nm}$.
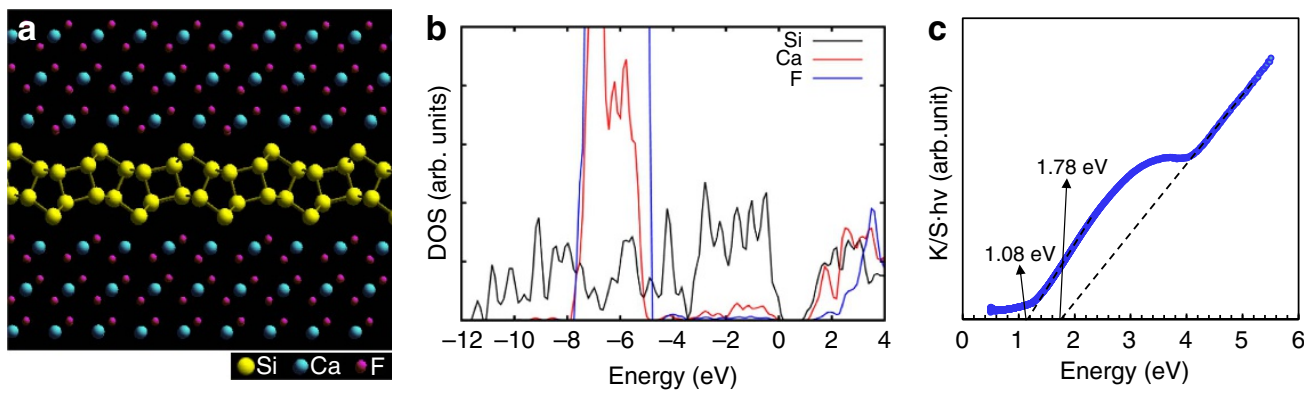

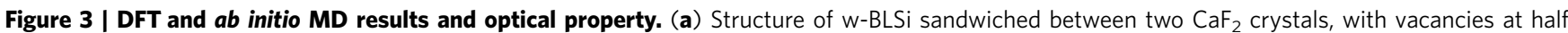
of the $\mathrm{F}$ sites on the interface; this structure was used to calculate the DOS and was obtained from the transformation of $\mathrm{i}-\mathrm{BLSi}$ in the $a b$ initio MD simulation and the subsequent quenching process (Supplementary Method). (b) Decomposed DOS for Si, Ca and F in w-BLSi displayed in a. (c) Plot of multiplication of the $\mathrm{K} / \mathrm{S}$ and energy as a function of energy for $\mathrm{CaSi}_{2} \mathrm{~F}_{1.8-2.3}$ consisting of $\mathrm{w}$-BLSi, trilayer silicene with dangling bonds and $\mathrm{F}$-terminated trilayer silicene. The absorption spectrum suggests two indirect gaps with values of 1.08 and $1.78 \mathrm{eV}$.

trilayer silicene and the $\mathrm{CaF}_{2}$ layer is inhibited ${ }^{19}$. From previous DFT results of monolayer and multilayer silicene terminated with atoms 37,38 , it is conjectured that the bandgap of F-terminated trilayer silicene would be $\sim 1 \mathrm{eV}$ within the framework of the DFT and Perdew, Burke and Ernzerhof (PBE) technique. It should be noted that DFT calculations using a standard generalized gradient approximation functional tend to underestimate the bandgap (roughly $\sim 2 / 3$ in crystal $\mathrm{Si}$ ). This indicates that the bandgap experimentally measured for the trilayer silicene should be $\sim 1.5 \mathrm{eV}$. Meanwhile, the bandgap for $\mathrm{w}$-BLSi, which is estimated to be $\sim 0.65 \mathrm{eV}$ in the DFT-PBE calculation, is expected to be $\sim 1 \mathrm{eV}$ in the experimental measurement. Therefore, the measured gaps were estimated such that the gaps of $\mathrm{w}$-BLSi and F-terminated trilayer silicene were 1.08 and $1.78 \mathrm{eV}$, respectively.

Transformation process from monolayer silicene to $\mathrm{w}$-BLSi. On the basis of the HAADF-STEM data, we discussed a model for the transformation process from a monolayer silicene in $\mathrm{CaSi}_{2}$ (Fig. 4a) to w-BLSi (Fig. 4f). When $\mathrm{F}^{-}$ions diffuse from the surface of a $\mathrm{CaSi}_{2}$ crystallite into the crystal along the Ca layer, 

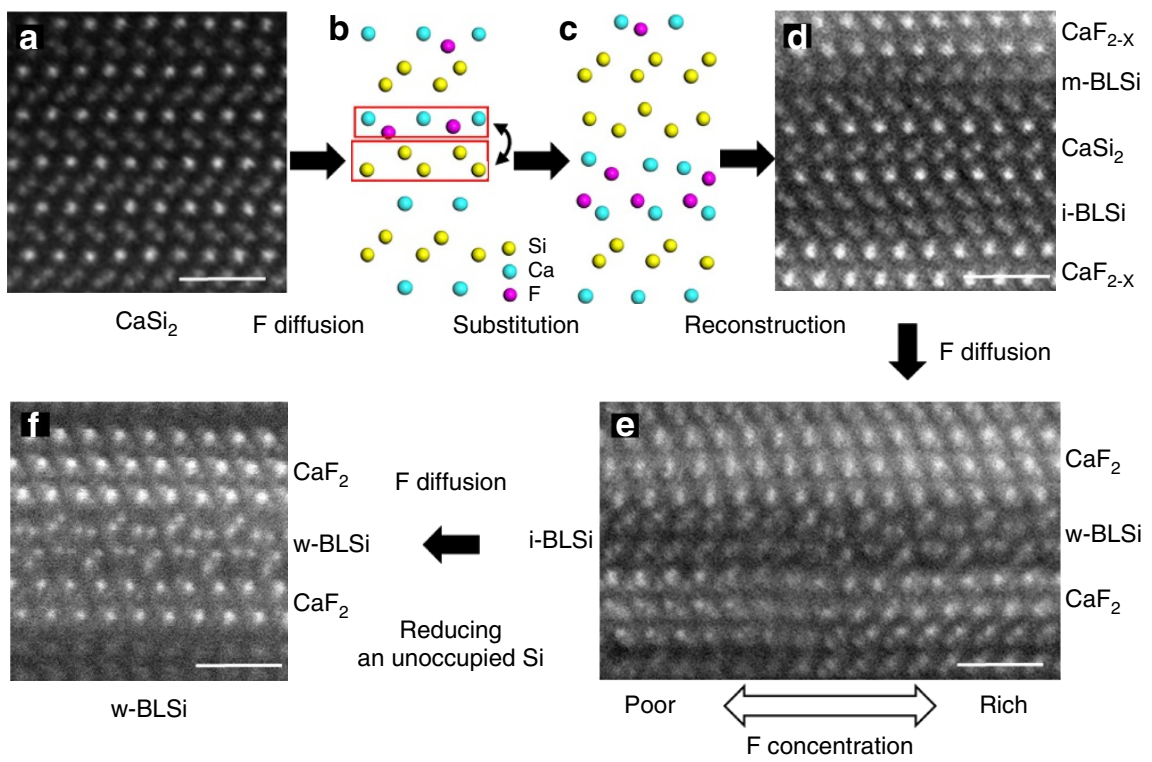

Figure 4 | A model for the transformation process from monolayer Si to w-BLSi. (a) and (d-f) HAADF-STEM image. (b,c) A schematic model. (a) Raw tr6 $\mathrm{CaSi}_{2}$. (b) $\mathrm{F}$ diffusion into $\mathrm{CaSi}_{2}$. (c) $\mathrm{A}$ random arrangement of $\mathrm{i}-\mathrm{BLSi}$ and bilayer $\mathrm{CaF}_{2-\mathrm{x}}$ in $\mathrm{CaSi}_{2}$. (d) $\mathrm{i}-\mathrm{BLSi}_{1}, \mathrm{CaF}_{2-x}$ and $\mathrm{CaSi} 2$ in a region with $\mathrm{CaSi}_{2} \mathrm{~F}_{\text {0.6-1.0. }}$ (e) $\mathrm{i}-\mathrm{BLSi}$ and $\mathrm{w}$-BLSi formed within the same layers in $\mathrm{CaSi}_{2} \mathrm{~F}_{0.6-1.0}$. (f) w-BLSi in $\mathrm{CaSi}_{2} \mathrm{~F}_{2.0}$. All scale bars, $1 \mathrm{~nm}$.

thin $\mathrm{CaF}_{2-\mathrm{x}}$ planar crystals are formed; as a result, anionic silicene layers assemble to reduce the number of unsaturated bonds beyond the Ca layer (Fig. 4b). During this movement, the Si covalent bonding network with honeycomb symmetry is broken and its arrangement consequently becomes random (Fig. 4c). As shown in Fig. 4d, two types of bilayer silicenes, i-BLSi and $\mathrm{m}-\mathrm{BLSi}$, which formed in the slit-like regions, as predicted by the MD calculation $^{22}$, co-exist with $\mathrm{CaSi}_{2}$ in the low F-concentration region. Both of these structures are stabilized as a result of charge transferred from the $\mathrm{Ca}$ atoms which saturate the silicon dangling bonds.

We analysed more than 200 STEM images of BLSi; w-BLSi was recognized at $\mathrm{F}$ concentrations surpassing that of $\mathrm{CaSi}_{2} \mathrm{~F}_{1.8}$. With increasing $\mathrm{F}$ concentration, the site occupancy of $\mathrm{F}$ concentration at the interface of the $\mathrm{CaF}_{2}$ planar crystal reached $\sim 0.5$, then $\mathrm{w}$-BLSi was formed by the change of ionic interactions among $\mathrm{Si}$, $\mathrm{Ca}$ and $\mathrm{F}$ (Fig. 3e,f). In this process, negatively charged $\mathrm{Si}$ atoms tend to lose their electrons, which makes i- (or m-) BLSi less stable because the 'capping' of the dangling bonds by extra electrons from $\mathrm{Ca}$ is reduced and the dangling bonds destabilize the $s p^{3}$ tetrahedral configuration. Thus, the anionic honeycomb structure of $\mathrm{i}^{-}$(or $\mathrm{m}-$ ) $\mathrm{BLSi}$ is transformed to $\mathrm{w}$-BLSi, which is approximately neutral because of the fluorination of the $\mathrm{Ca}$ cation.

\section{Discussion}

We focused on calcium-intercalated silicene $\left(\mathrm{CaSi}_{2}\right)$ and discovered a strategy for transforming monolayer silicene into a novel bilayer silicene (w-BLSi). From HAADF-STEM images, we observed that $\mathrm{w}$-BLSi was formed between the planar crystals of $\mathrm{CaF}_{2}$ and contained four-, five- and six-membered silicon rings, although w-BLSi consists of only Si atoms exhibiting tetrahedral coordination. Compared with monolayer silicene, the number of unsaturated silicon bonds in w-BLSi decreased to $25 \%$ of the unit cell. The transformation process from monolayer silicene in $\mathrm{CaSi}_{2}$ to $\mathrm{w}$-BLSi was estimated from HAADF-STEM data. When $\mathrm{F}^{-}$ ions diffuse into the $\mathrm{CaSi}_{2}$ crystal along the Ca layer, thin $\mathrm{CaF}_{2-\mathrm{x}}$ planar crystals and two types of bilayer silicenes (i-BLSi and $\mathrm{m}$-BLSi) are formed, following breakage of the $\mathrm{Si}$ covalent bonding monolayer network. Both of these $\mathrm{Si}$ structures were stabilized as a result of charge transferred from the $\mathrm{Ca}$ atoms which saturate the silicon dangling bonds. With increasing $\mathrm{F}$ content, i- (or m-) BLSi is transformed to w-BLSi. Additionally, the structure possesses an indirect bandgap of $1.08 \mathrm{eV}$ in contrast to monolayer silicene, which is a zero-gap semiconductor.

\section{Methods}

Synthesis of $\mathrm{CaSi}_{2} \mathbf{F}_{\mathbf{X}}$ compound. $\mathrm{CaSi}_{2}$ single-crystal grains $(0.1 \mathrm{~g})$ were reacted with $5 \mathrm{ml}$ of ionic liquid [BMIM] [BF $\left.{ }_{4}\right]$ (1-butyl-3-methylimidazolium tetrafluoroborate) at $300^{\circ} \mathrm{C}$ for $15 \mathrm{~h} . \mathrm{BF}_{4}^{-}$decomposed into $\mathrm{F}^{-}$during annealing, and the $\mathrm{CaSi}_{2}$ crystal was changed to $\mathrm{CaSi}_{2} \mathrm{~F}_{\mathrm{X}}$ compounds $(0 \leq \mathrm{X} \leq 2.3)$ through the diffusion of $\mathrm{F}^{-}$(Fig. 1a,b). More details are given in Supplementary Method.

Chemical composition analysis. The chemical compositions of the $\mathrm{CaSi}_{2} \mathrm{~F}_{\mathrm{X}}$ domains were determined by electron probe microanalyser (EPMA) with a wave dispersion system (JEOL JXA-8200), an accelerating voltage of $10 \mathrm{kV}$, a specimen current of $50 \mathrm{nA}$, and an electron irradiation area of $5 \mu \mathrm{m} \varphi$. Single-phase $\mathrm{CaF}_{2}$ and $\mathrm{Si}$ crystals were used as the standard for quantitative composition analysis of $\mathrm{Ca}$, $\mathrm{F}$ and $\mathrm{Si}$. EPMA line analyses were performed with $5 \mu \mathrm{m}$ steps from the edge to the inside of the $\mathrm{CaSi}_{2} \mathrm{~F}_{\mathrm{X}}$ crystallites cross-sectioned parallel to the $\mathrm{CaSi}_{2}[001]$ direction.

TEM/STEM analysis. HAADF-STEM observations $\mathrm{s}^{28-30}$ and STEM energydispersive X-ray spectroscopy (EDX) analyses were performed with a Titan ${ }^{3}$. G2 60-300 electron microscope (FEI, $\mathrm{Cs}=156 \mathrm{~nm}$ ) operated at $300 \mathrm{kV}$.

HAADF-STEM imaging was capable of providing an atomic-scale Z-contrast image associated with the heavier constituent elements. The annular detector was set to collect the electrons scattered at angles between 50.5 and $200 \mathrm{mrad}$. High-resolution transmission electron microscopy observations were obtained with a JEM-2000EX electron microscope (JEOL, $\mathrm{Cs}=0.7 \mathrm{~mm}$ ) operating at $200 \mathrm{kV}$. TEM specimens of $\mathrm{CaSi}_{2} \mathrm{~F}_{\mathrm{X}}$ were detected with five different $\mathrm{F}$ concentration ranges $\left(\mathrm{CaSi}_{2} \mathrm{~F}_{0.6^{-1}, 0}, \mathrm{CaSi}_{2} \mathrm{~F}_{16}, \mathrm{CaSi}_{2} \mathrm{~F}_{1.8}, \mathrm{CaSi}_{2} \mathrm{~F}_{20}\right.$ and $\left.\mathrm{CaSi}_{2} \mathrm{~F}_{23}\right)$ by using the FIB microsampling method ${ }^{39}$. The atomic positions in the w-BLSi crystal and the interface structure were characterized by comparing the HAADF-STEM image contrasts with simulated contrasts calculated by the multi-slice method using MacTempasX.

Computational method. DFT and ab initio MD calculations were performed to calculate the DOS and to examine the structural stability of BLSi using the Vienna $A b$ initio Simulation Package (ref. 40). The projector augmented wave method ${ }^{41}$ and generalized gradient approximation with the exchange and correlation functions of PBE were employed ${ }^{42}$. A plane-wave basis set with an energy cutoff of $400 \mathrm{eV}$ was used with $\Gamma$-point sampling in the Brillouin zone. To model the BLS systems observed in our experiments, two-layer $\mathrm{Si}$ structures were sandwiched by $\mathrm{CaF}_{2}$ crystal domains, each consisting of three sets of $\mathrm{CaF}_{2}$ layers, with or without the F-site vacancy at the $\mathrm{Si} / \mathrm{CaF}_{2}$ interfaces. The DOS for the w-BLSi was calculated for the structure obtained after the quenching process (shown in Fig. 3a) following the $300 \mathrm{~K}$ run. More details are given in Supplementary Method. 
Optical reflectivity. Diffuse reflectance spectra were obtained for the $\mathrm{CaSi}_{2} \mathrm{~F}_{1.8-2.3}$ composition powder sample using a spectrophotometer (JASCO V-670)

The diffuse reflectance spectra were processed under the Kubelka-Munk formalism, and the bandgaps were determined using a plot of the multiplication of the K/S and energy. More details are given in Supplementary Methods.

\section{References}

1. Novoselov, K. S. et al. Electric field effect in atomically thin carbon films. Science 306, 666-669 (2004)

2. Novoselov, K. S. et al. Two-dimensional gas of massless dirac fermions in graphene. Nature 438, 197-200 (2005).

3. Zhang, Y., Tan, Y. W., Stormer, H. L. \& Kim, P. Experimental observation of the quantum hall effect and berry's phase in graphene. Nature 438, 201-204 (2005).

4. Novoselov, K. S. et al. Room-temperature quantum Hall Effect in Graphene. Science 315, 1379 (2007).

5. Takeda, K. \& Shiraishi, K. Theoretical possibility of stage corrugation in Si and Ge analogs of graphite. Phy. Rev. B 50, 14916 (1994).

6. Cahangirov, S., Topsakal, M., Aktürk, E., Şahin, H. \& Ciraci, S. Two- and onedimensional honeycomb structures of silicon and germanium. Phys. Rev. Lett. 102, 236804 (2009)

7. Lebègue, S. \& Eriksson, O. Electronic structure of two-dimensional crystals from ab initio theory. Phys. Rev. B 79, 115409 (2009).

8. Vogt, P. et al. Silicene: Compelling experimental evidence for graphenelike two-dimensional silicon. Phys. Rev. Lett. 108, 155501 (2012).

9. Fleurence, A. et al. Experimental evidence for epitaxial silicene on diboride thin films. Phys. Rev. Lett. 108, 245501 (2012).

10. Okamoto, H. et al. Silicon nanosheets and their self-assembled regular stacking structure. J. Am. Chem. Soc. 132, 2710-2718 (2010).

11. Sugiyama, Y. et al. Synthesis and optical properties of monolayer organosilicon nanosheets. J. Am. Chem. Soc. 132, 5946-5947 (2010).

12. Okamoto, H., Sugiyama, Y. \& Nakano, H. Synthesis and modification of silicon nanosheets and other silicon nanomaterials. Chem. Eur. J. 17, 9864-9887 (2011).

13. Tao, L. et al. Silicene field-effect transistors operating at room temperature. Nat. Nanotechnol. 10, 227-231 (2015).

14. Morishita, T., Spencer, M. J. S., Kawamoto, S. \& Snook, I. K. A new surface and structure for silicene: polygonal silicene formation on the $\mathrm{Al}(111)$ surface. J. Phys. Chem. C 117, 22142-22148 (2013).

15. Gao, J. \& Zhao, J. Initial geometries, interaction mechanism and high stability of silicene on $\mathrm{Ag}(111)$ surface. Sci. Rep. 2, 861 (2012).

16. Cahangirov, S. et al. Electronic structure of silicene on $\operatorname{Ag}(111)$ : strong hybridization effects. Phys. Rev. B 88, 035432 (2013).

17. Noguchi, E. et al. Direct observation of dirac cone in multilayer silicene intercalation compound $\mathrm{CaSi}_{2}$. Adv. Mater. 27, 856-860 (2015).

18. Yaokawa, R., Nakano, H. \& Ohashi, M. Growth of $\mathrm{CaSi}_{2}$ single phase polycrystalline ingots using the phase relationship between $\mathrm{CaSi}_{2}$ and associated phases. Acta Mater. 81, 41-49 (2014).

19. Kokott, S., Pflugradt, P., Matthes, L \& Bechstedt, F. Nonmetallic substrates for growth of silicene: an ab initio prediction. J. Phys. Condens. Matter 26, 185002 (2014).

20. Morishita, T., Spencer, M. J. S., Russo, S. P., Snook, I. K. \& Mikami, M. Surface reconstruction of ultrathin silicon nanosheets. Chem. Phys. Lett. 506, 221-225 (2011).

21. Sakai, Y. \& Oshiyama, A. Structural stability and energy-gap modulation through atomic protrusion in freestanding bilayer silicene. Phy. Rev. B 91, 201405(R) (2015).

22. Morishita, T., Nishio, K. \& Mikami, M. Formation of single- and double-layer silicon in slit pores. Phy. Rev. B 77, 081401(R) (2008).

23. Bai, J., Tanaka, H. \& Zeng, X. C. Graphene-like bilayer hexagonal silicon polymorph. Nano Res 3, 694-700 (2010).

24. Johnston, J. C., Phippen, S. \& Molinero, V. A single-component silicon quasicrystal. J. Phys. Chem. Lett. 2, 384-388 (2011).

25. Pflugradt, P., Matthes, L. \& Bechstedt, F. Unexpected symmetry and AA stacking of bilayer silicene on $\mathrm{Ag}(111)$. Phys. Rev. B 89, 205428 (2014).

26. Guo, Z.-X. \& Oshiyama, A. Structural tristability and deep Dirac states in bilayer silicene on $\operatorname{Ag}(111)$ surfaces. Phys. Rev. B 89, 155418 (2014).

27. Cahangirov, S. et al. Atomic structure of the $\sqrt{3} \times \sqrt{3}$ phase of silicene on Ag(111). Phys. Rev. B 90, 035448 (2014).

28. Pennycook, S. J. \& Jesson, D. E. High-resolution incoherent imaging of crystals. Phys. Rev. Lett 64, 938-941 (1990).

29. Pennycook, S. J. \& Jesson, D. E. High-resolution z-contrast imaging of crystals. Ultromicroscopy 37, 14-38 (1991).
30. Pennycook, S. J. \& Jesson, D. E. Atomic resolution Z-contrast imaging of interfaces. Acta Mater. 40, S149-S159 (1992).

31. Huang, S., Kang, W. \& Yang, L. Electronic structure and quasiparticle bandgap of silicene structures. Appl. Phys. Lett. 102, 133106 (2013).

32. Lee, P. A., Said, G., Davis, R. \& Lim, T. H. On the optical properties of some layer compounds. J. Phys. Chem. Solids 30, 2719-2729 (1969).

33. Mak, K. F., Lee, C., Hone, J., Shan, J. \& Heinz, T. F. Atomically thin $\mathrm{MoS}_{2}$ : a new direct-gap semiconductor. Phys. Rev. Lett. 105, 136805 (2010).

34. Gaiser, C. et al. Band-gap engineering with $\mathrm{HfS}_{\mathrm{X}} \mathrm{Se}_{2} \square \mathrm{x}$. Phys. Rev. B 69, 075205 (2004).

35. Bianco, E. et al. Stability and exfoliation of germanane: a germanium graphane analogue. ACS Nano 7, 4414-4421 (2013).

36. Kamal, C., Chakrabarti, A., Banerjee, A. \& Deb, S. K. Silicene beyond mono-layers - different stacking configurations and their properties. J. Phys. Condens. Matter 25, 085508 (2013).

37. Gao, N., Zheng, W. T. \& Jiang, Q. Density functional theory calculations for two-dimensional silicene with halogen functionalization. Phys. Chem. Chem. Phys. 14, 257-261 (2012)

38. Morishita, T. et al. First-principles study of structural and electronics properties of ultrathin silicon nanosheets. Phys. Rev. B 82, 045419 (2010).

39. Kirk, E. C. G., Williams, D. A. \& Ahmed, H. Cross-sectional transmission electron microscopy of precisely selected regions from semiconductor devices. Inst. Phys. Conf. Ser. 100, 501-506 (1989).

40. Kresse, G. \& Furthmüller, J. Efficiency of ab-initio total energy calculations for metals and semiconductors using a plane-wave basis set. Comput. Mater. Sci. 6, 15-50 (1996)

41. Blöchl, P. E. Projector augmented-wave method. Phys. Rev. B 50, 17953 (1994)

42. Perdew, J. P., Burke, K. \& Ernzerhof, M. Generalized gradient approximation made simple. Phys. Rev. Lett. 77, 3865 (1996).

\section{Acknowledgements}

This work was supported in part by PRESTO, the Japan Science and Technology Agency, and by a Grant-in-Aid for Scientific Research from the Ministry of Education, Culture, Sports, Science and Technology (MEXT), Japan. HAADF-STEM observations in this work were supported by the 'Nanotechnology Platform' of MEXT, Japan, at the Center for Integrated Nanotechnology Support, Tohoku University. The computations were undertaken with the assistance of resources from the National Computational Infrastructure (NCI), which is supported by the Australian Government, the Pawsey Supercomputing Centre with funding from the Australian Government and the Government of Western Australia, the Multi-modal Australian ScienceS Imaging and Visualisation Environment (MASSIVE) and the Victorian Partnership for Advanced Computing Limited (VPAC Ltd) through the V3 Alliance, Australia, and at the computational facilities at the Research Center for Computational Science, National Institute of Natural Sciences, and at the Research Institute for Information Technology, Kyushu University, Japan. We thank Mr Y. Yagi for the EPMA measurements, Prof. S. Yamanaka and Dr Y. Takeda for fruitful discussion.

\section{Author contributions}

R.Y. and H.N. conceived the idea. R.Y. and T.O. designed the experiments. R.Y. synthesized the $\mathrm{CaSi}_{2}$ single crystals and $\mathrm{CaSi}_{2} \mathrm{~F}_{\mathrm{X}}$ compounds. Y.H. performed the HAADF-STEM observations and the EDX analyses. T.M. and M.J.S.S. performed the theoretical work. R.Y. and T.O. characterized the w-BLSi. R.Y., T.O., T.M. and H.N. wrote the manuscript. All the authors have read the manuscript and agree with its content.

\section{Additional information}

Supplementary Information accompanies this paper at http://www.nature.com/ naturecommunications

Competing financial interests: The authors declare no competing financial interests.

Reprints and permission information is available online at http://npg.nature.com/ reprintsandpermissions/

How to cite this article: Yaokawa, R. et al. Monolayer-to-bilayer transformation of silicenes and their structural analysis. Nat. Commun. 7:10657 doi: 10.1038/ncomms10657 (2016).

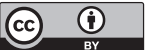

This work is licensed under a Creative Commons Attribution 4.0 International License. The images or other third party material in this article are included in the article's Creative Commons license, unless indicated otherwise in the credit line; if the material is not included under the Creative Commons license, users will need to obtain permission from the license holder to reproduce the material. To view a copy of this license, visit http://creativecommons.org/licenses/by/4.0/ 